\title{
Axillofemoral bypass with local anesthesia: a way forward to enable limb salvage in high-risk patients
}

\author{
Abdullah Al-Wahbi \\ Department of Vascular Surgery, \\ King Abdulaziz Medical City, Vascular \\ Surgery Division, Riyadh, Saudi Arabia
}

This article was published in the following Dove Press journal:

Local and Regional Anesthesia

9 October 2010

Number of times this article has been viewed

\begin{abstract}
For an active, ambulant patient with critical, lower limb ischemia, amputation can lead to a poor quality of life. A small group of older people with critical limb ischemia are considered at high risk for revascularization under conventional anesthesia owing to their comorbid conditions. In these cases, when endovascular therapy is not an option, the decision to amputate or revascularize presents a dilemma, especially in ambulant patients. In this article, we present 2 cases in which the individuals had diabetic foot gangrene, rest pain, and multiple comorbidities, and were unfit to undergo conventional anesthesia. In addition, they had severe aortoiliac occlusive disease, which cannot be managed by endovascular methods. Both patients were living independently and were ambulant before their foot ulcer and ischemia. They underwent an axillofemoral bypass under local anesthesia. The postoperative course was uneventful. After a 3-year follow-up, both patients continue to be ambulant and have no complaints. With selective use of local anesthetic techniques, surgical teamwork to shorten the procedure time, and close meticulous postoperative care, an axillofemoral bypass can enable limb salvage for ambulant patients who are considered unfit for conventional anesthesia.
\end{abstract}

Keywords: limb salvage, axillofemoral bypass, local anesthesia, high-risk patients

\section{Introduction}

The procedure used to treat critical lower limb ischemia is mainly dependent on a patient's fitness for conventional anesthesia, especially when the arterial pathology is not amenable to endovascular management. Unfortunately, a few older patients with critical lower limb ischemia have associated comorbidities that make them unfit for conventional anesthesia. ${ }^{1-3}$ Usually, those patients are treated by amputation, despite the possible negative effects on the independence and quality of life of the patient. In this article, we present a study of 2 individuals who had diabetic foot gangrene, rest pain, and multiple comorbidities that made them unfit to undergo general anesthesia (GA). Axillofemoral (Ax-Fem) bypass was performed under local anesthesia (LA). In both patients, limb salvage was successfully achieved. After a 3-year follow-up, both patients maintain full ambulatory function and patent grafts. We reviewed the literature on this subject and found no reports of performing an Ax-Fem bypass with only LA.

\section{Case I}

The patient was a 53-year-old man who was a heavy smoker and a diabetic on insulin. He had coronary artery disease and severe peripheral arterial disease. He presented with severe bilateral, lower limb ischemia, with absent femoral and distal pulses in both lower limbs. The right and left ankle brachial indices (ABIs) were 0.4 and 0.3 , 
respectively. An angiogram revealed complete occlusion of the infrarenal aorta and iliac arteries, with patent femoral and distal arteries bilaterally (Figure 1). His infrainguinal arteries were normal in appearance. The patient had undergone a coronary artery bypass 5 years earlier and then multiple cardiac catheterizations and stenting for recurrent chest pain. A cardiology evaluation that included a stress test was aborted because the patient developed severe chest pain with significant changes in the electrocardiogram during the procedure. Subsequent nuclear studies confirmed fixed cardiac defects.

\section{Case 2}

The patient was a 63-year-old man who was hypertensive and a diabetic on insulin. He had coronary artery disease that had been treated with multiple cardiac catheterizations and stenting and had severe bilateral peripheral artery disease. He was being conservatively treated for bilateral carotid artery stenosis. A year earlier, he presented with bilateral lower limb ischemia and left foot toes infection. Angiogram showed severe aortoiliac occlusive disease. He had bilateral common iliac angioplasty and stenting, followed by amputation of his infected toes. His left foot healed completely. On this admission, he presented with sepsis that had resulted from wet gangrene of his right forefoot. He had absent femoral and distal pulses with bilateral ABIs of 0.3. After an emergency metatarsal amputation under ankle block, he was transferred

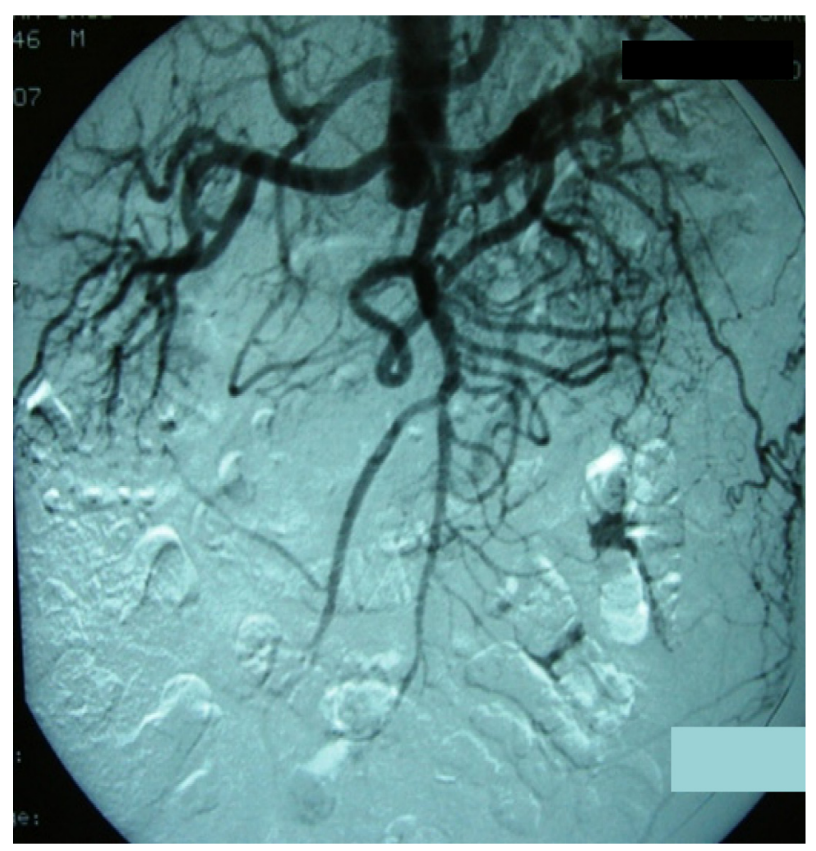

Figure I Angiogram of the case I patient showing complete occlusion of infrarenal aorta. to intensive care unit (ICU) for postoperative monitoring and discharged to the ward after the third day. Computerized tomography angiogram and angiography revealed complete occlusion of the infrarenal aorta and iliac arteries, with patent femoral and distal arteries bilaterally (Figure 2). A cardiac evaluation revealed that he had an ejection fraction of $25 \%-30 \%$ and left ventricular dilatation with multiple wall abnormalities.

The cardiologist labeled both patients as at a high risk of mortality if they were to undergo a procedure under GA, as determined by the American College of Cardiology/American Heart Association risk stratification guidelines. ${ }^{1}$ In addition, the anesthesiologist labeled them as ASA 4 class. The patients were thin, ambulatory, and worked at desk jobs to support their families. Both patients refused to consider amputation as an option and demanded to be discharged if there were no other alternatives. The vascular surgery, anesthesia, cardiology, and ICU consultant teams discussed the cases and decided to perform an Ax-Fem bypass under LA with close perioperative and postoperative intensive monitoring. After the procedure and its risks were discussed with the patients, they consented for the bypass to be performed.

\section{Procedures}

The first patient weighed $65 \mathrm{~kg}$, was $170 \mathrm{~cm}$ tall, and had a body mass index (BMI) of 22.5. The second patient weighed $62 \mathrm{~kg}$, was $167 \mathrm{~cm}$ tall, and had a BMI of 22.2. Both had normal liver and renal functions. The same procedural steps and anesthesia were used for both patients. Central vein and radial arterial lines were inserted. Exposure and control of the right axillary and the 2 femoral arteries were performed simultaneously after slow infiltration of a 1:1 mixture of $2 \%$ lignocaine and $0.25 \%$ bupivicaine. This same mixture was used for the entire procedure. Epinephrine was not added to

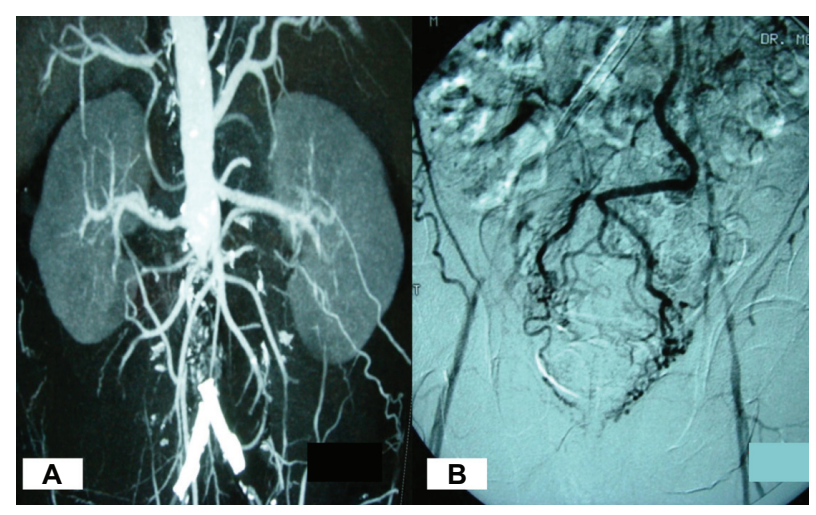

Figure 2 Computerized tomography angiogram A) and angiography B) of case 2 patient showing complete occlusion of infrarenal aorta with reconstitution at femoral vessels, and occluded iliac stents $\mathbf{B}$ ). 
the anesthesia because it could have caused vasoconstriction of the femoral or axillary arteries. An 8-mm ringed Gortex graft with a premade femoral bifurcation was used. After the graft was anastomosed to the axillary artery, the subcutaneous tissue of the tunnel area was infiltrated with LA in a $2 \mathrm{~cm}$ width from the groin to the axillary wound. The Atrium vascular tunneler system was chosen for both patients because it had several advantages. It had a pointed tip and a long handle, providing excellent control and easy passage through the subcutaneous tissue. In addition, the tip had different sizes to accommodate the graft diameter used. The graft was tunneled using a tunneler with an $8 \mathrm{~mm}$ tip, followed by bilateral femoral anastomosis. One patient (case 1) had a total of $80 \mathrm{~mL}$ of LA, whereas the other patient (case 2) had $100 \mathrm{~mL}$. As determined by each patient's weight, the total amount of LA, $100 \mathrm{~mL}, 2 \%$ lignocaine $(325 \mathrm{mg}$ ) or $0.25 \%$ bupivicaine $(43 \mathrm{mg})$, did not exceed the maximum dose. Neither patient developed the signs of LA toxicity, which can include lightheadedness, tinnitus, or circumoral and tongue numbness. Because both patients were awake and did not complain of any problems, the anesthetist did not have the local anesthetic plasma levels measured. The operative procedure time was 110-140 minutes, and the patients were hemodynamically stable during the perioperative period. A small dose of Midazolam was used in the second patient when he became restless for a short period of time, which accounts for the longer operative time and the greater amount of LA $(100 \mathrm{~mL})$. At the end of the procedure, both patients had palpable pedal pulses. They were transferred to the ICU and remained stable there for 3 days, then transferred to the ward. Ten days after the procedure, the first patient was ambulatory and discharged. He was examined during a close outpatient follow-up. The amputation stump of the second patient started to heal with good granulation tissue (Figure 3). Two weeks after the procedure, he underwent a skin graft under tumescent anesthesia. The stump healed completely,

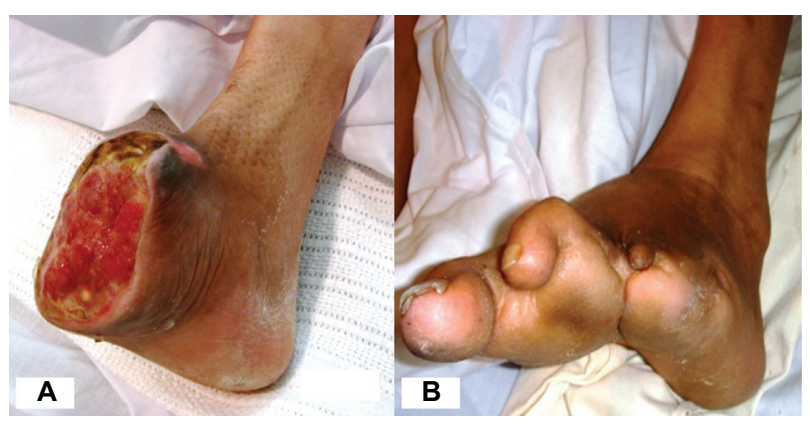

Figure 3 Case 2, right foot stump with good granulation tissue 10 days post bypass A). The left foot with previous toes amputation B). and he started ambulating with custom-made shoes. He was discharged 4 weeks after the bypass procedure.

\section{Discussion}

Medical advances are enabling people with chronic diseases to live longer, and the majority of them maintain a good quality of life. ${ }^{4}$ Because of the high prevalence of diabetes mellitus and peripheral arterial disease, foot complications and critical lower limb ischemia are among the most common reasons for hospital admissions. Most of these patients have chronic diseases (eg, coronary artery disease, hypertension, renal, or respiratory impairment) that give them a poor life expectancy and place them at a high risk for surgical revascularization under conventional anesthesia. ${ }^{3}$ The majority of perioperative and postoperative mortalities are due to cardiac complications. ${ }^{2,3,5-8}$ In patients who are bedridden and terminally ill, and have extensive foot or leg gangrene, with obliteration of all normal runoff arteries in the calf or foot as assessed by good quality angiography or surgical exploration, ${ }^{3,6}$ the decision to amputate is usually straightforward. The dilemma comes when the patient is ambulant, independent, and at high risk for conventional anesthesia. It is universally agreed that limb salvage in an ambulant patient is important for maintaining independence and quality of life. ${ }^{4,9}$ In addition, major amputations have a high mortality, morbidity, and health care cost. ${ }^{4}$ Choosing the type of anesthesia depends on a number of factors. For regional or LA, the nature of the surgical procedure, the patient's comorbidities (eg, mental status), and the skill of the anesthesia and surgical teams should be considered. ${ }^{10}$ The decision must be made on case-by-case basis. The cardiologists' preoperative assessment must be integrated by the anesthesia and surgical teams. ${ }^{1}$ Epidural anesthesia has less perioperative and postoperative complications than GA but is associated with a risk of hypotension that may not be tolerated by certain high-risk patients. ${ }^{6,9}$ In addition, vascular patients are often on chronic use of acetylsalicylic acid, clopidogrel, or coumadin. As well, intravenous heparin is usually administered intraoperatively. All of these can lead to epidural hematoma. ${ }^{10} \mathrm{LA}$, nerve blocks, and sedation for infrainguinal bypasses were reported to have acceptable complication rates in high-risk patients. ${ }^{1,6,8}$ In patients with aortoiliac occlusive disease not amenable to endovascular intervention, aortobifemoral bypass (ABF) is the standard surgical procedure because of its proven long-term effectiveness., ${ }^{5,11,12}$ When an ABF procedure is not an option for those who are at high risk, have a hostile abdomen, or have lesions not amenable to endovascular management, an extra-anatomic bypass, mainly Ax-Fem, is an acceptable alternative..$^{511-16}$ Although Ax-Fem 
bypass is less invasive, and requires a shorter procedure time than $\mathrm{ABF}$, when GA is used it has a reported mortality of $1.7 \%-16 \%$ depending on the indication of the procedure. ${ }^{5}$ The Ax-Fem bypass carries a peculiar challenge when compared with infrainguinal bypass because nerve blocks are not helpful in the area of the axillary dissection and graft tunneling. On reviewing the literature, we found that only 1 study used LA for an Ax-Fem bypass. However, it was performed with femoral nerve blocks and sedation. ${ }^{9}$ In our report, only LA was used in 1 patient, and 1 dose of sedation was used in the other.

In conclusion, we believe that in certain high-risk patients who are ambulant, independent, and with critical lower limb ischemia due to aortoiliac occlusive disease not amenable to endovascular management, Ax-Fem bypass under LA is a viable option for limb salvage. Patient selection, teamwork between the different medical teams, the presence of 2 vascular surgeons' teams operating simultaneously to shorten the procedure time, and perioperative and postoperative intensive care monitoring are mandatory considerations.

\section{Disclosure}

Presented at the 11th Annual Congress of the Asian Society for Vascular Surgery (ASVS 2010) and at the 4th annual meeting of the Federation of Vascular Surgery Societies (WFVS).

The author declares no financial disclosures or conflicts of interest in this work.

\section{References}

1. Fleisher LA, Beckman JA, Brown KA, et al. ACC/AHA 2007 guidelines on perioperative cardiovascular evaluation and care for noncardiac surgery. J Am Col Cardiol. 2007;50:159-242.

2. Back MR, Leo F, Cuthbertson D, Johnson BL, Shamesmd ML, Bandyk DF. Long-term survival after vascular surgery: specific influence of cardiac factors and implications for preoperative evaluation. $J$ Vasc Surg. 2004;40(4):752-760.
3. Varu VN, Hogg ME, Kibbe MR. Critical limb ischemia. J Vasc Surg. 2010;51:230-241.

4. Pomposelli FB, Arora S, Gibbons GW, et al. Lower extremity arterial reconstruction in the very elderly: successful outcome preserves not only the limb but also residential status and ambulatory function. J Vasc Surg. 1998;28:215-225.

5. Schneider JR, McDaniel MD, Walsh DB, et al. Axillofemoral bypass: outcome and hemodynamic results in high-risk patients. J Vasc Surg. 1992;15(6):952-962.

6. Barkmeier LD, Hood DB, Sumner DS, et al. Local anesthesia for infrainguinal arterial reconstruction. Am J Surg. 1997;174(20):202-204.

7. Miyajima S, Shiria A, Yamamoto S, et al. Risk factors for major limb amputations in diabetic foot gangrene patients. Diabetes Res Clin Pract. 2006;71:272-279.

8. Jin F, Chung F. Minimizing perioperative adverse events in the elderly. Br J Anaesth. 2001;87:608-624.

9. Mackay CA, Razik W, Simms MH. Local anesthetic for lower-limb revascularization in high-risk patients. Br J Surg. 1997;84(8):1096-1098.

10. Lumb AB. Anesthesia for vascular surgery on extremities. Anaest Intensive Care Med. 2007;8(6):255-259.

11. Passman MA, Taylor LM, Moneta GL, et al. Comparison of axillofemoral and aortofemoral bypass for aortoiliac occlusive disease. J Vasc Surg. 1996;23(2):263-271.

12. Onohara T, Komori K, Kume M, et al. Multivariate analysis of long-term results after an axillobifemoral and aortobifemoral bypass in patients with aortoiliac occlusive disease. J Cardiovasc Surg. 2000;41(6):905-910.

13. Naylor AR, Ah-see AK, Engeset J. Axillofemoral bypass as a limb salvage procedure in high risk patients with aortoiliac disease. Br J Surg. 1990;77(6):659-661.

14. Harrington ME, Harrington EB, Haimov M, Schanzer H, Jacobson JH II. Axillofemoral bypass: compromised bypass for compromised patients. J Vasc Surg. 1994;20(2):195-201.

15. O'Toole DP, Cuningham AJ. Regional anesthesia for major vascular surgery. Yale J Biol Med. 1993;66:447-456.

16. Hung Lau, Stephen Wing-Keung Cheng. Axillofemoral bypass for limb salvage. Ann Coll Surg Hong Kong. 2008;4(4):149-153.

17. Tsui BC, Wagner A, Finucane B. Regional anesthesia in the elderly: a clinical guide. Drugs Aging. 2004;21(14):859-910.
Local and Regional Anesthesia

\section{Publish your work in this journal}

Local and Regional Anesthesia is an international, peer-reviewed, open access journal publishing on the development, pharmacology, delivery and targeting and clinical use of local and regional anesthetics and analgesics. The journal welcomes submitted papers covering original research, basic science, clinical studies, reviews \& evaluations,

\section{Dovepress}

guidelines, expert opinion and commentary, case reports and extended reports. The manuscript management system is completely online and includes a very quick and fair peer-review system, which is all easy to use. Visit http://www.dovepress.com/testimonials.php to read real quotes from published authors. 